\title{
A DISTRIBUTED MICROCOMPUTER TELEMETRY SYSTEM FOR SPACECRAFT APPLICATIONS*
}

\author{
Donald D. Lord \\ Member of the Technical Staff \\ Jet Propulsion Laboratory \\ California Institute of Technology \\ Pasadena, California 91103
}

\begin{abstract}
Three significant difficulties exist in using a set of microcomputers (or any computers) as an integral part of an on-board telemetry system. The difficulties arise in providing for:

a) the precise timing required by a telemetry system, including accurate time-tagging of data samples;

b) the interconnection of several (many) computers in a controlled, organized, understandable manner;

c) the capability to allow computer controlled science instruments to operate relatively autonomously with minimum software interactions with other computers.
\end{abstract}

The three items listed above are desirable features and are achievable with proper telemetry system architecture designs. This paper will address each of the three items and present an architecture that provides the desired features.

\section{INTRODUCTION}

The Caltech Jet Propulsion Laboratory (JPL) is conducting an advanced development effort to design and implement a spacecraft distributed computer Command and Data Handling $(\mathrm{C} \& \mathrm{DH})$ subsystem. The effort is being sponsored under a NASA Research and Technology Objective and Plan (RTOP) and has been on-going for about four years. The C\&DH subsystem includes, as a major part, functions performed by spacecraft telemetry systems, and is a vital element in the NASA End-to-End Data System (NEEDS) program.

\footnotetext{
* This paper presents the results of one phase of research carried out by the Jet Propulsion Laboratory, California Institute of Technology, under Contract No. NAS 7-100, sponsored by the National Aeronautics and Space Administration.
} 
An objective of the program is to demonstrate flight readiness of the C\&DH subsystem by flying it, along with a complement of science instruments, on a Space Shuttle flight in 1982. Progress to date has included the design, fabrication, and successful operation of a breadboard distributed computer C\&DH subsystem. ${ }^{1,2,3}$.

\section{DISTRIBUTED MICROCOMPUTER TELEMETRY SYSTEM DESIGN DIFFICULTIES}

\section{Basic Causes of Design Difficulties}

The most common source of problems associated with using microcomputers (or any computer) as an integral part of an on-board telemetry system is related to timing accuracy. Computers, being software controlled devices, do not normally provide the same fixed, precise, synchronized, unalterable operation that a hard-wired system does. However, with carefully thought out architecture designs, the precise timing and synchronization required by telemetry systems can be provided for in computer based systems.

A second source of design problems is associated with understanding and controlling the hardware and software interactions that can arise when several (many) computers are connected together and must communicate with one another. Unexpected interactions, conflicts, priorities, etc. must all be resolved in an organized manner whenever they occur. The approach taken in the architecture design being presented herein is to avoid the need to resolve these types of problems by not allowing them to occur in the first place. Obviously not all conflicts and interactions can be avoided - some must be resolved-but those that effect critical timing accuracy can be.

\section{Three Significant Design Difficulties}

Three timing related design difficulties are being addressed herein. Architecture designs are presented that resolve the problems and provide the same timing accuracy as hardwired systems do. The difficulties arise in providing for:

a) the precise timing required by a telemetry system, including accurate time tagging of data samples;

b) the interconnection of several (many) computers in a controlled, organized, understandable manner;

c) the capability to allow computer controlled science instruments to operate relatively autonomously with minimum software interactions with other computers. 


\section{SOLUTIONS TO DISTRIBUTED MICROCOMPUTER TELEMETRY SYSTEM DESIGN DIFFICULTIES}

\section{Precise Time Tagging of Data Samples}

Hardwired telemetry systems provide precisely timed sequences for collecting telemetry data. Particular measurements are sampled at specific times in the commutation cycle, and the timing cannot change. Time information (absolute time or spacecraft time) is inserted into the telemetry stream at specific points in the commutation cycle, making it possible to accurately determine when data samples were taken-within a microsecond or so.

In general it is not necessary to know sample times as accurately as a few microseconds. There are times, however, when it does become important; such as when correlating data between two or more instruments or when trying to resolve cause and effect situations.

Commutation cycles in computer based systems are software controlled and as such are subject to timing variations caused by such things as servicing interrupts, updating program parameters, and execution of subroutines. Unless the program runs through the exact same set of instructions (or at least instructions of equivalent time duration) each time through the commutation cycle, variations in data sample times will occur. One method of avoiding the timing variations is to actually count the number of instructions in the worst case (longest) program loop that could ever be encountered and then add dummy instructions in the shorter loops to make them all equal in length. This method has been used in the past and works, but does make software generation (and modification) very difficult.

The method employed for avoiding timing variations in the taking of telemetry samples in the C\&DH design is indicated in Figure 1. This design avoids the need to count instructions, yet provides equivalent accuracy in time tagging of data. The scheme employs the use of a hardware clock to provide simultaneous real time interrupts (RTI) to all computers in the system to initiate software routines. Assuming an RTI as a reference point, typical software routines then address a particular telemetry measurement sometime (anytime) before the next (second) RTI. Exactly on the second RTI, analog and/or digital data is strobed into sample and hold circuits. Digital data is then available for loading into telemetry (memory) buffers anytime between the second and third RTIs. In the case of analog data the period between the second and third RTIs is used for A/D conversions, making the converted data available one RTI period later (between the third and fourth RTIs). 


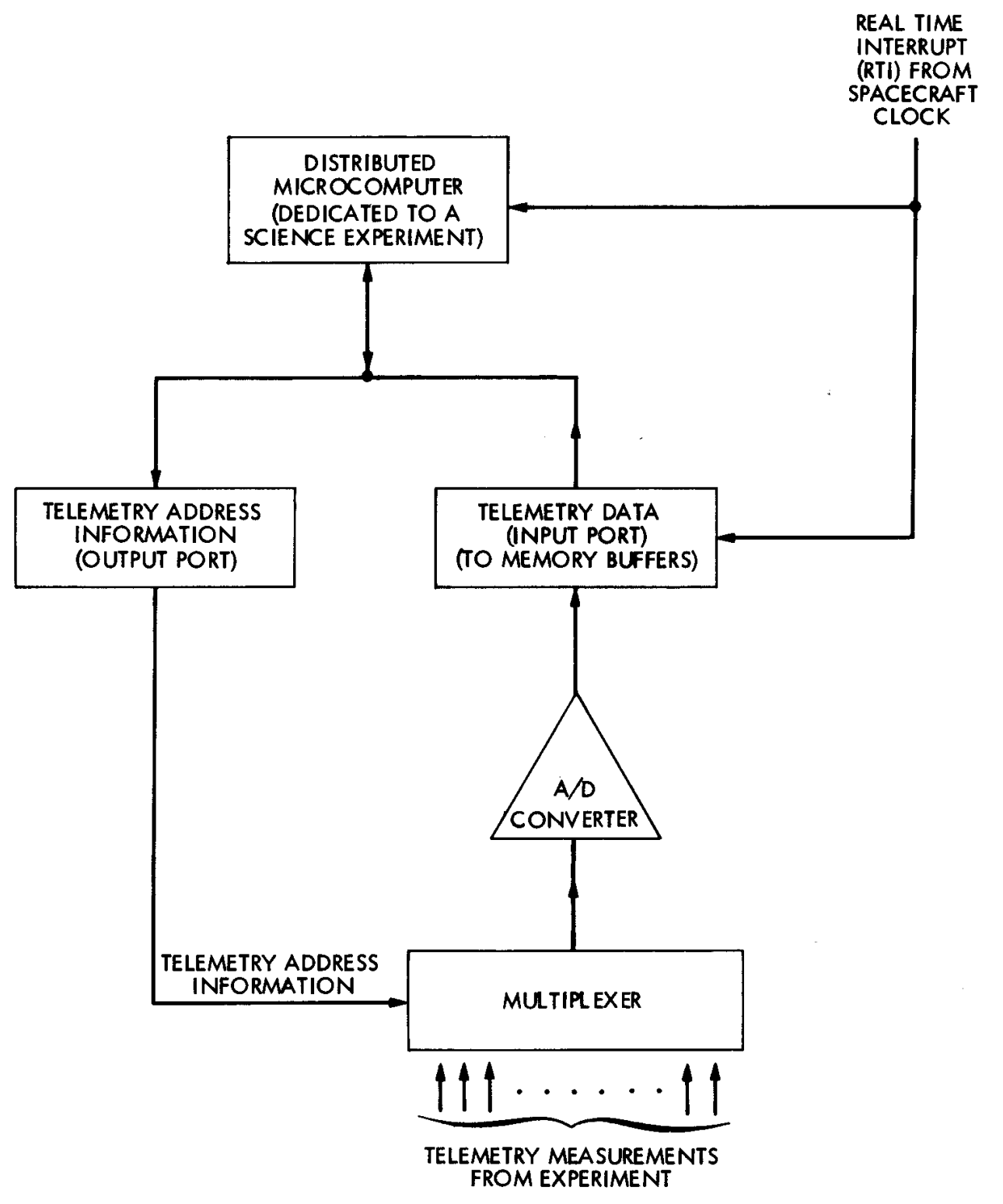

Figure 1 - Gathering of Telemetry Data

The scheme may sound somewhat complicated, but the simple timing diagram of Figure 2 shows it to be very straight forward while providing known, unalterable, accurate time tagging of telemetry samples. It also allows the software controlling the commutation cycle, long periods of time (one RTI period) to get things done, with the only constraint being that it be done prior to the following RTI. Typical RTI periods are two to ten milliseconds with the current operating breadboard at JPL using 2.5 milliseconds. Any RTI period will provide the same microsecond accuracy on time tagging of individual data samples. Note that while microsecond accuracy is maintained, timing resolution is reduced to the granularity of milliseconds (one RTI period). 


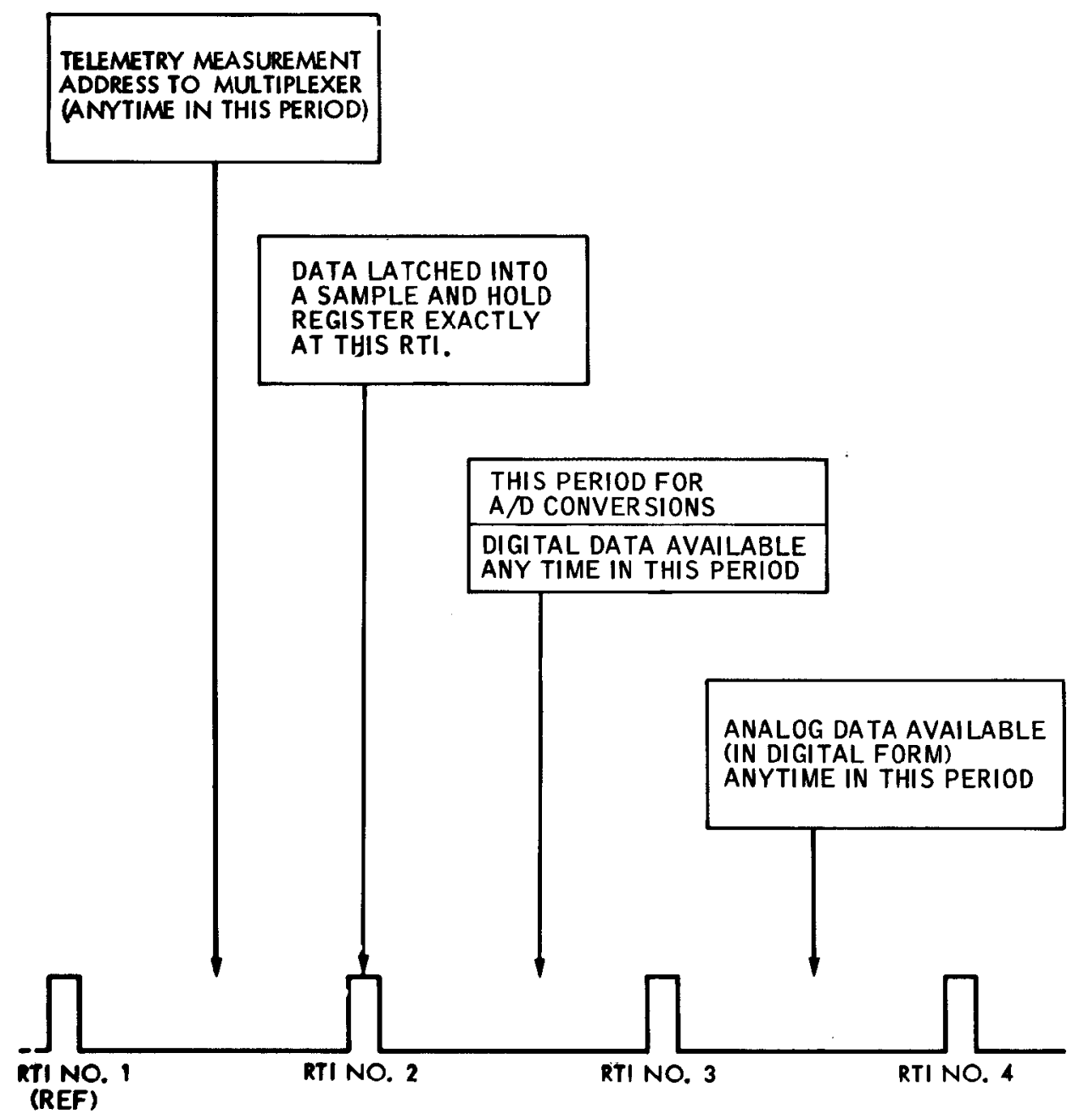

Figure 2 - Timing of Telemetry Gathering Function

Higher data rates (higher than one telemetry sample per RTI) are handled through the use of direct memory access (DMA) ports. Typical operation involves setting up the DMA port (specifying the starting address of telemetry measurements and the number of measurements to be taken) sometime during an RTI period. A high rate telemetry clock synchronized to the RTI clock would then latch the data into memory buffers at the rate of one measurement per clock cycle, starting at the next (second) RTI. Software development is kept simple (since a complete RTI period is allowed for initializing the DMA port) and the microsecond time tagging accuracy is maintained (since data is latched into buffers by a hardware clock).

\section{Interconnection of Several (Many) Computers}

The telemetry system being discussed is a distributed computer system. The data gathering is actually distributed among a number of computers, each with its own commutation cycle, data storage buffers, and specified set of measurements to be sampled. A computer 
based High Level Module (HLM) controls the formation of the final telemetry stream by accessing individual computer's data storage buffers at the appropriate time and inserting them into the telemetry stream. Figure 3 shows the basic architecture of the C\&DH (telemetry) system.

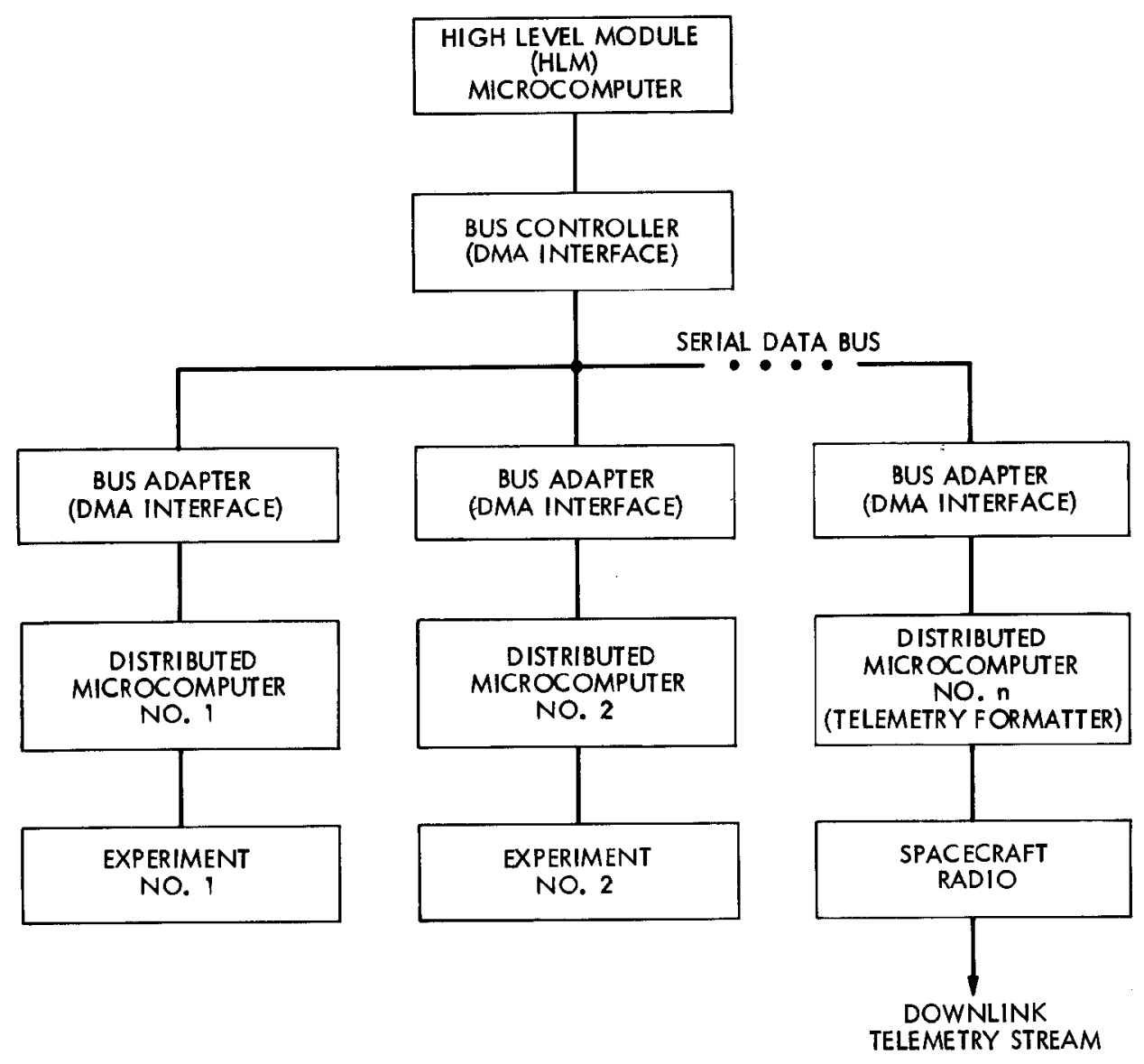

Figure 3 - C\&DH Subsystem (Telemetry System) Architecture

The interconnection of all computers in the system provides a controlled, organized, understandable, interface. This is accomplished by tieing all computers together on a common serial data bus, but allowing only the High Level Module the capability to initiate transfers. Having all transfers controlled from a single source eliminates conflicts or the need to resolve priorities on the use of the bus.

Interfacing to the bus itself is accomplished through the use of external DMA hardware. Transfers are set up in the applicable computer's DMA hardware by the High Level Module without interferring with any on-going program . Once the DMA ports are set up in both the sending and receiving units the transfer takes place directly from one computer's memory to another's memory (DMA) and still does not disturb on-going programs. The advantage of DMA transfers is that they are controlled by external hardware, do not require the use of the processor of the computers involved in the transfer, 
and do not interfere with on-going programs. In effect the computers involved in the transfer do not even realize it is occurring except as it modifies memory locations and software programs that may involve the use of those locations.

Precise timing of data transfers is maintained through the use of the RTI. Transfers are set up during one RTI period, and executed during the next. Additional detail on timing of data transfers is available in references. ${ }^{1,2,3}$.

\section{Autonomous Operation of Science Instruments}

Many science experiments flying on future spacecraft will include a dedicated microcomputer as an integral part of the experiment. The distributed computers discussed herein for the telemetry system can serve as dedicated experiment control computers as well as performing the data gathering function. When used in this dual fashion, the computer becomes part of the C\&DH subsystem (which includes the telemetry system) since commanding, instrument control, and data handling/gathering of the experiment are all managed by its own dedicated microcomputer.

Science experiments should also be relatively autonomous to simplify testing and interfacing to the spacecraft system. The interface to the spacecraft data system (telemetry system) should be transparent to them so that they could operate during testing exactly as they would when connected to the spacecraft. Having this autonomy has a significant beneficial affect on both cost and complexity associated with experiment/spacecraft integration. The experiment is controlled, operated and monitored identically during lab testing as it is in spacecraft system operation.

Figure 4 shows the architecture design that allows a science experiment to have its own dedicated microcomputer, be relatively autonomous, maintain precise timing accuracy, and have a transparent interface to the spacecraft data system. (In reality no interface can be completely transparent but it is transparent in that it allows the experiment hardware and software to operate identically in lab testing as it does in spacecraft system operation.) The experiment is autonomous since all commands, including data (telemetry) gathering commands, must be issued by its own dedicated computer. System type commands (whether coming from test equipment or from the spacecraft C\&DH system) modify memory as necessary to cause the experiment computer to issue the command to its own experiment. No outside direct access to the experiment is allowed (except for monitoring operation during testing) thereby maintaining the instrument autonomy.

The gathering of experiment data (telemetry data) is also an autonomous operation since it is performed by and stored in the dedicated experiment computer. 

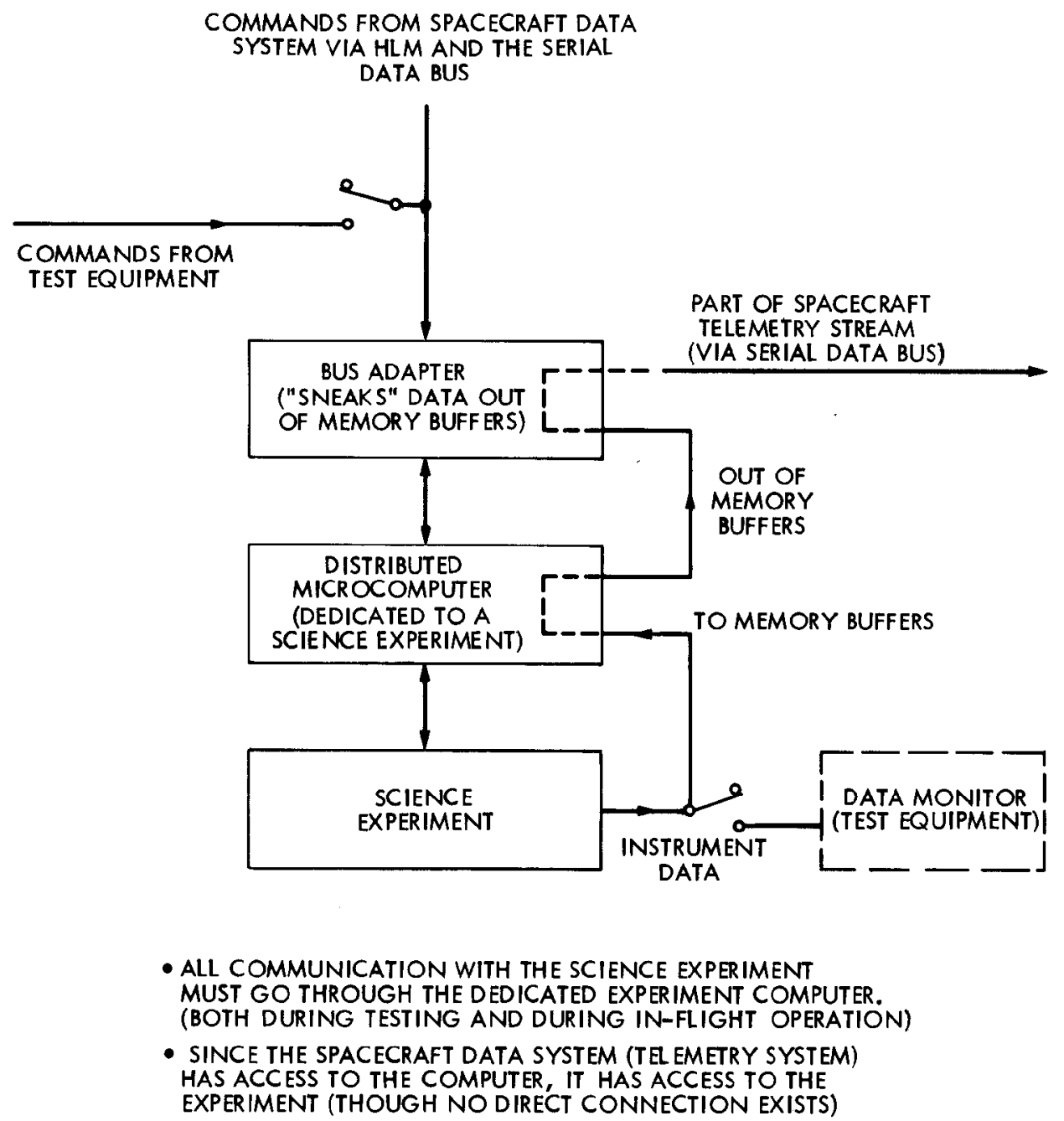

Figure 4 - Autonomous Instrument Operation

Each dedicated experiment computer keeps track of time (spacecraft time or real time depending on the implementation of the master spacecraft clock) within itself. A software clock in each computer is initialized (resynchronized) periodically by the HLM using the master spacecraft clock as a source of time information. Software in each distributed computer causes its clock to increment once each RTI, thereby causing all computers to maintain identical software clock information internally. Autonomy is maintained In that no computer has to go outside its own memory to get spacecraft time information, yet all computers are synchronized to a single master clock.

\section{RELIABILITY CONSIDERATIONS}

Spacecraft telemetry systems must be among the most reliable systems on the spacecraft, since a loss of telemetry results in a loss of all meaningful science data. 
In the C\&DH subsystem, reliability is achieved through the use of flight qualified components, worst case design techniques, elimination of single point failure modes and adding of redundant elements at critical points.

Redundant elements that may be added include microcomputers, data busses, interface circuits, or individual components as dictated by the reliability requirements of a particular mission. The basic system architecture remains unchanged since the C\&DH subsystem is designed in a modular fashion to allow for adding additional modules to provide increased reliability. Additional information may be obtained from the references . ${ }^{1,3}$.

\section{CONCLUSIONS}

Distributed computer telemetry systems, as an integral part of a spacecraft Command and Data Handling (C\&DH) subsystem, have been demonstrated to have significant advantages over hardwired (or single computer programmable) telemetry systems in certain applications. Typical applications include spacecraft systems that require telemetry data from a variety of experiments and also have significant computational tasks to perform. Combining telemetry data gathering functions with science experiment controlling functions allows an experiment to gain autonomy through the use of a dedicated computer (controller). Autonomous operation is important as spacecraft systems become more complex since it minimizes interractions and integration problems with other elements of the spacecraft. Carefully thought out architecture designs can simplify data systems (telemetry system) operation while maintaining the precise, accurate, timing and synchronization that is required.

\section{ACKNOWLEDGEMENTS}

The author wishes to thank D.A. Rennels, M.E. Ebersole, and H.F. Lesh for valuable discussions contributing to the content of this paper.

\section{REFERENCES}

1. Rennels, D.A., Riis Vestergaard, B., and Tyree, V.C., "The Unified Data System: A Distributed Processor Network for Control and Data Handling on a Spacecraft," Proc. IEEE National Aerospace and Electronics Conference, NAECON, Dayton, Ohio, May 1976.

2. Lesh, R., and Lecoq, P., "Software Techniques for a Distributed Real-Time Processing System," Proc. IEEE National Aerospace and Electronics Conference, NAECON, Dayton, Ohio, May 1976.

3. Rennels, D.A., "A Distributed Microprocessor System for Spacecraft Control and Data Handling," Proc. MIDCON Electronic Show and Convention, Chicago, Illinois, November 1977. 